\title{
The exchange rates of amide and arginine guanidinium CEST in the mouse brain
}

Kexin Wang ${ }^{1,3^{*}}$, Ran Sui ${ }^{1,3^{*}}$, Lin Chen ${ }^{1,2,4}$, Yuguo $\mathrm{Li}^{1,2}$, Jiadi $\mathrm{Xu}^{1,2}$

1. F.M. Kirby Research Center for Functional Brain Imaging, Kennedy Krieger Research Institute, Baltimore, MD, USA

2. Russell H. Morgan Department of Radiology and Radiological Science, Johns Hopkins University School of Medicine, Baltimore, MD, USA

3. Department of Biomedical Engineering, Johns Hopkins University, Baltimore, MD, USA

4. Department of Electronic Science, Fujian Provincial Key Laboratory of Plasma and Magnetic Resonance, Xiamen University, Xiamen, China

*These authors contributed equally to this work

Corresponding Author:

Jiadi Xu, Ph.D.

Kennedy Krieger Institute

Johns Hopkins University School of Medicine

707 N. Broadway

Baltimore, MD, 21205

E-mail: jxu37@jh.edu

Tel: 443-923-9572

Fax: 443-923-9505

Grant support from NIH: P41EB031771, R01HL149742, and R21AG074978.

Running title: amide and arginine proton exchange rates 


\begin{abstract}
:
Purpose: To develop a pipeline for measuring the exchange rates and concentrations of in vivo excgangeable protons, and to demonstrate this for the amide and arginine (Arg) guanidinium (Guan) protons in mobile proteins in the mouse brain.

Methods: An ultra-short echo (UTE) CEST sequence with a continuous wave presaturation (preRadCEST) was applied to acquire Z-spectra with robustness to motion and physiological fluctuations. AmideCEST and Arginine guanCEST (ArgCEST) were extracted and their proton concentrations and exchange rates obtained using a two-step multi- $\mathrm{B}_{1}$ Bloch fitting approach that included the semisolid macromolecular background. To minimize contamination from the amine protons from creatine and phosphocreatine, ArgCEST measurements were performed on the Guanidinoacetate N-methyltransferase deficiency $\left(\mathrm{GAMT}^{-/}\right)$mouse characterized by low creatine and phosphocreatine concentrations in the brain.

Results: For the amideCEST proton pool, the exchange rate and concentrations were found to be $59.6 \pm 9.0 \mathrm{~s}^{-1}$ and $41.7 \pm 7.0 \mathrm{mM}$, respectively, with the maximum signal observed at $\mathrm{B}_{1}=0.8 \mu \mathrm{T}$. For the ArgCEST proton, the guanidinium exchange these were $70.1 \pm 5.5 \mathrm{~s}^{-1}$ and $10.1 \pm 1.3 \mathrm{mM}$, respectively, with the maximum effect observed at $\mathrm{B}_{1}=0.9 \mu \mathrm{T}$. The current study suggests that the inverse $\mathrm{pH}$ dependence in GuanCEST of brain is led by the CrCEST component, not ArgCEST. Conclusion: The current pipeline is expected to have general use for in vivo CEST quantitation and optimization of visible CEST resonances.
\end{abstract}

\title{
Key Words:
}

Chemical exchange saturation transfer (CEST), amide, arginine, guanidinium, exchange rate, concentration, presaturation radial CEST (preRadCEST). 


\section{Introduction}

Chemical exchange saturation transfer (CEST) MRI is a molecular imaging method that can significantly enhance the sensitivity of detecting low concentrations of proteins and metabolites through the water signal (1-3). Among many endogenous CEST contrasts, amide protons (amide proton transfer, APT, or amideCEST) in mobile proteins have been intensively studied. AmideCEST signal has a well-defined lineshape, which stands out in the crowded in vivo Z-spectrum $(4,5)$. It is noteworthy that amideCEST should be distinguished from APT-weighted MRI, which also focuses on the peak at $3.5 \mathrm{ppm}$ but is obtained by asymmetry analysis of the Zspectrum and thus includes multiple other effects including those from relayed NOEs (rNOEs) and asymmetry in the semisolid magnetization transfer contrast (MTC) (6). Guanidinium (Guan) CEST was reported in creatine (Cr) phantoms (7-9), muscle (10-13), and brain (14), the Zspectrum of which at $2 \mathrm{ppm}$ also includes the phosphocreatine ( $\mathrm{PCr}$ ) and the guanidinium protons of arginine groups in endogenous mobile proteins (5,15-18). Since for proteins the only guanidinium group exists in Arginine (Arg) groups, we name the GuanCEST signal from proteins as ArgCEST, while $\mathrm{Cr}$ as CrCEST. AmideCEST and ArgCEST are among a few CEST contrasts that can be extracted with high confidence from the crowded in vivo Z-spectrum because of their distinguishable peaks at multiple MRI field strengths. The optimum acquisition and quantification of amideCEST and ArgCEST contrasts usually requires knowledge of their exchange rates. Particularly, the exchange rate of the ArgCEST proton pool is critical in the understanding of the $\mathrm{pH}$ and $\mathrm{B}_{1}$ dependence of signal at $2 \mathrm{ppm}(15,19-21)$. For most metabolite CEST studies, the exchange rates can be measured in phantom solution under physiological conditions, such as glutamate (22), D-glucose $(23,24), \mathrm{Cr}(9)$, and $\mathrm{PCr}(9,25,26)$. However, this method is not possible for both amideCEST and ArgCEST since these resonances reflect the composite effect of multiple unidentified proteins. An initial attempt to determine the amide proton exchange rate was carried out in the rat brain with a water-exchange filter (WEX) NMR sequence, giving a value of $28.6 \mathrm{~s}^{-1}$ (27). Recent studies, including frequency labeled exchange transfer MRI (28) and fingerprinting CEST method, suggested a much higher exchange rate (around 200-400 s-1) (29), while another group reported as 34.6-47.9 $\mathrm{s}^{-1}$ with fingerprinting $(30,31)$. Hence, it is worth revisiting the amideCEST exchange rate with recent technology and also fill in the long-standing blank of the ArgCEST exchange rate. 
The direct measurement of exchange rates of amideCEST and ArgCEST from the brain Zspectrum is hampered by three factors. Firstly, the acquisition of Z-spectrum needs correction for the noise and artifacts introduced by physiological motions $(32,33)$ and/or MRI instability. A large variation is observed in the Z-spectrum when the saturation field strength in Hertz is comparable to the $\mathrm{B}_{0}$ fluctuation in the mouse brain caused by the respiration and brain movements (34). This can be partially improved using the motion-insensitive CEST MRI methods developed recently, such as the self-gated ultra-short echo time (UTE) CEST technique (35-37), or using navigators to correct for frequency fluctuations and motion. (38-40) Secondly, the extraction of both amideCEST and ArgCEST signals from in vivo Z-spectrum is challenging. Multi-pool Lorentzian line-shape fitting does not function well for the amide and guanidinium resonances (41-45), due to their overlap with amineCEST, PCr amide, and amide/aromatic rNOE signals, especially for guanidinium. Fortunately, recent $\mathrm{pH}$-dependent studies on animal stroke models at both high $(4,15,21)$ and low MRI fields (18) have suggested that both amideCEST and ArgCEST show discernible peaks with smooth backgrounds in the brain Z-spectrum. These peaks can be well extracted and quantified using a recently proposed polynomial and Lorentzian line-shape fitting (PLOF) approach $(17,20,25,34)$. Another challenge for the ArgCEST measurement is the contamination from $\mathrm{Cr}$ guanidinium and $\mathrm{PCr}$ amide peaks around $2 \mathrm{ppm}(16,17)$. This problem can be solved by performing the ArgCEST assessment using a Guanidinoacetate Nmethyltransferase deficiency $\left(\mathrm{GAMT}^{-/}\right)$mouse model that has low $\mathrm{Cr}$ and $\mathrm{PCr}$ concentrations in the brain $(46,47)$. Thirdly, the quantitation of the CEST exchange rate in the brain with the QUESP (the quantification of exchange rate using varying saturation power) $(48,49)$ and Omega plot $(50$ 52) methods needs to account for the strong MTC and direct saturation backgrounds in tissues. To overcome all of the challenges mentioned above, we developed a two-step multi-B Bloch- $_{1}$ McConnell (BM) fitting approach for both signal extraction (background removal) and quantification. The idea is similar to that behind PLOF CEST, but strictly follows the BM equations for fitting the MTC background and the CEST peaks. The approach was first validated in a creatine $(\mathrm{Cr})$ phantom mixed with cross-linked bovine serum albumin (BSA) and then applied in vivo for the amideCEST with wild type (WT) mouse and ArgCEST using $\mathrm{GAMT}^{-/}$mouse brains. 


\section{Methods}

\section{Presaturation Radial CEST sequence}

The CEST pulse sequence with a radial readout $(35-37,53)$ was composed of a train of interleaved Gaussian saturation pulses and 2D-UTE modules. As demonstrated in both brain (35) and liver CEST studies (36), a radial acquisition scheme is inherently less sensitive to motion artifacts. However, the steady-state UTE-CEST is difficult for CEST quantification as it is challenging to model the pulsed CEST signal. Therefore, we modified the sequence to perform continuous wave (CW) CEST labeling at the beginning followed by a train of 2D UTE readout modules as shown in Fig. 1, which is dubbed presaturation radial CEST (preRadCEST).

\section{CEST extraction and quantification}

Three pools, i.e., water, magnetization transfer contrast (MTC) and CEST, were assumed for the BM equations. Here, MTC is a combined pool from both macromolecules and other CEST components. For the macromolecular MTC proton pool, the transverse relaxation time can be neglected due to its extremely short $\mathrm{T}_{2}(\sim 10 \mu \mathrm{s})$ (54). Then, the MTC components are determined by three parameters (supplemental material Eq. S4): the MTC exchange rate $k_{\mathrm{MTC}}$, the back exchange rate from MTC to water $k_{M T C} \cdot f_{M T C}$, and the $R_{1 M T C}+R_{M T C} \cdot R_{1 M T C}$ is typically much lower than $R_{M T C}$ and has minimum impact on the fitting, and was set to $1 \mathrm{~s}^{-1}$ here. $T_{2 \mathrm{MTC}}$ usually determines the MTC lineshape in the conventional Super-Lorentzian or Gaussian assumptions for the biological tissues $(55,56)$. In current study, a linear lineshape was used for the mixed MTC and CEST background as we fit only a small range of the Z-spectrum $(<4 \mathrm{ppm})$. Then, $T_{2 \mathrm{MTC}}$ has no impact on the fitting results and was set to $10 \mu$ s. The detailed BM fitting is provided in the supporting information and the Matlab code for the BM fitting will be made available at https://github.com/jiadixu/Two-step-Bloch-Fitting after the paper is accepted. The Z-spectra acquired with different $\mathrm{B}_{1}$ values are fitted simultaneously, i.e., group BM fitting. Two steps of the fitting are performed as below:

1. The MTC and water background spectrum were fitted by excluding the data points for amideCEST (or ArgCEST) with varying parameters, $T_{2 a p p}, C_{M T C}^{0}$ and $C_{M T C}^{1}$, for each Zspectrum. Here, $T_{2 a p p}$, is a parameter including water transverse relaxation and the broadening caused by the fast-exchanging protons. The offset was fixed at $3.5 \mathrm{ppm}$ (amideCEST) and 2.0 ppm (ArgCEST), while $T_{1}$ of the amide and ArgCEST pool was set to $1 \mathrm{~s}$. The $T_{l w}$ values 
measured in both phantom and mouse brain were fixed for the group fitting. The fitting was achieved by minimizing the root mean square (RMS) of the difference between our fitting and experimental data. For amideCEST and ArgCEST, background fitting ranges of 2.0-5.5 ppm and 1.0-3.5 ppm were used, excluding the relevant signal ranges of $[3.2,4.0] \mathrm{ppm}$ and $[1.6,2.6]$ ppm, respectively.

2. The extraction and fitting of amideCEST and ArgCEST signals were achieved by fixing the background signal obtained in step 1 , i.e., the $T_{1 \mathrm{w}}, T_{2 a p p}, C_{M T C}^{0}, C_{M T C}^{1}$ values. The $T_{2 \text { amide, }} k_{\text {amide }}$ and $f_{\text {amide }}$ (or $T_{2 \text { Guan, }} k_{\text {Guan }}$ and $f_{\text {Guan }}$ ) values were varied in the amideCEST (or ArgCEST) fitting to minimize the RMS of the difference between our fitting and experimental data. Either amideCEST or ArgCEST signal can be obtained by subtracting the background signal from the CEST signals at 3.5 and 2.0 ppm respectively.

\section{Phantom Validation}

The two-step BM fitting method was validated on a $\mathrm{Cr}(50 \mathrm{mM})$ solution mixed with $15 \%$ cross-linked BSA (CrCrossBSA) in phosphate buffered saline (PBS), titrated to pH 7.7. BSA crosslinking was achieved using a $25 \mu \mathrm{L}$ glutaraldehyde solution. The exchange rate of the CrCrossBSA phantoms was measured on a Bruker 17.6T NMR spectrometer using the selective inversion recovery method detailed in the supplemental material (13).

\section{MRI Experiments}

Animal procedures were approved by the Johns Hopkins University Animal Care and Use Committee. Six C57BL/6 mice (age: 3 months) and four GAMT ${ }^{-/-}$mice (age: 3 months) were used. All MRI experiments were performed on a horizontal bore 11.7 T Bruker Biospec system (Bruker, Ettlingen, Germany). A $72 \mathrm{~mm}$ quadrature volume resonator was used as a transmitter, and a 2x2 phased array was used as a receiver for both phantoms and animals. Animals were anesthetized using $2 \%$ isoflurane in medical air, followed by $1 \%$ to $1.5 \%$ isoflurane for maintenance. The $\mathrm{B}_{0}$ field over the image slice was adjusted to second order for animal using field-mapping and shimmed on the water linewidth for phantoms. $\mathrm{T}_{1}$-weighted maps were acquired using the same geometry and spatial resolution as CEST MRI, employing a RAREVTR sequence (RARE with variable $\mathrm{TR}=0.5,1,1.5,2,3.5,5,8 \mathrm{~s})$. 
The pre-saturation time was set to $2 \mathrm{~s}$ for all CEST experiments. Seven $\mathrm{B}_{1}$ values were collected for the CrCrossBSA phantom $(0.4,0.6,0.8,1.0,1.2,1.4$ and $1.6 \mu \mathrm{T})$, while eight $\mathrm{B}_{1}$ values were used for the mouse brain $(0.4,0.5,0.6,0.8,1.0,1.2,1.4$ and $1.6 \mu \mathrm{T})$. The frequency was swept from 2.0 to $5.5 \mathrm{pm}$ with an increment of $0.1 \mathrm{ppm}$ for the amideCEST. This same frequency step size was applied for the ArgCEST from $1.0 \mathrm{ppm}$ to $3.5 \mathrm{ppm}$, except for a smaller increment of $0.05 \mathrm{ppm}$ between $1.4 \mathrm{ppm}$ and $3.0 \mathrm{ppm}$. For phantom studies, the frequency was swept from 0.5 to $3.5 \mathrm{ppm}$ with $0.1 \mathrm{ppm}$ increment. The excitation pulse for the 2D-UTE radial readout was $0.4 \mathrm{~ms}$ with a flip angle of 10 degrees. After each saturation pulse, one radial spoke was collected with TR2 $=6 \mathrm{~ms}$. In the current study, single-slice CEST images were collected with a total acquisition time of $900 \mathrm{~ms}$. The nominal Cartesian matrix size was set to $96 \times 96$ with the slice thickness $=2 \mathrm{~mm}$. Before performing experiments on the $\mathrm{GAMT}^{-/-}$mice, in vivo MRS was performed on the mouse brain to confirm the low concentration of $\mathrm{Cr} / \mathrm{PCr}$ (Supporting Information Figure S1).

\section{Results}

\section{Phantom validation experiments}

One challenging part in the BM fitting is the great number of parameters that participate, while they are interacting with each other. We used a CrCrossBSA phantom as ground truth to validate the fitting method. The typical MRS spectrum of CrCrossBSA is plotted in the inset of Fig. 2a. The Guan exchange rate at room temperature and $\mathrm{pH} 7.7$ was determined to be $106 \pm 8 \mathrm{~s}^{-}$ ${ }^{1}$ by fitting the selective inversion curves of the Guan peak (Fig. 2a).

CEST experiments were performed on the same phantom at room temperature with the preRadCEST sequence. To examine the proper selection of $k_{\mathrm{MTC}}, f_{\mathrm{MTC}}$ and $T_{2 \mathrm{MTC}}$ values on the MTC background fitting. The MTC background fitting in the step one was evaluated with different $k_{\mathrm{MTC}}, T_{2 \mathrm{MTC}}$ and $f_{\mathrm{MTC}}$ values while $T_{2 a p p}, C_{M T C}^{0}$ and $C_{M T C}^{1}$ varied for each $\mathrm{B}_{1} \mathrm{Z}$-spectrum (Figs. 2bd). The goodness-of-fit was evaluated by the $R^{2}$ coefficient. The result suggested that the fitting is insensitive to the $f_{\mathrm{MTC}}$ and $k_{\mathrm{MTC}}$ when $f_{\mathrm{MTC}}>8 \%$ and $k_{\mathrm{MTC}}>11 \%$. Hence, typical in vivo $k_{\mathrm{MTC}}=$ $20 \mathrm{~s}^{-1}, f_{\mathrm{MTC}}=10 \%$ were fixed in our study, which are representative in vivo MTC parameters determined by on- and off-resonance variable delay multi-pulse (VDMP) methods $(57,58)$ as well as previous MTC studies (59-65) 
The typical Z-spectrum as well as the BM fitting curves are plotted in Fig. 2e. The fitting curves matched the observed Z-spectrum for all $\mathrm{B}_{1}$ values $\left(\mathrm{R}^{2}>0.995\right)$. From the two-step $\mathrm{BM}$ fitting, CrCEST exchange rate was determined to be $104.7 \pm 6.0 \mathrm{~s}^{-1}$, concentration $=46.8 \pm 5 \mathrm{mM}$ and $T_{2 \text { Guan }}=18.3 \pm 2 \mathrm{~ms}$. The exchange rate and concentration matched well with the measured rate from the inversion experiment $\left(106 \pm 8 \mathrm{~s}^{-1}\right)$ and the prepared concentration $(50 \mathrm{mM})$. The fitting results for all parameters are listed in the supporting information Table S1. The CrCEST signal as a function of $B_{1}$ plotted in Fig. $2 \mathrm{f}$ shows typical buildup and decay pattern with the peak at $0.8 \mu \mathrm{T}$.

\section{AmideCEST in the WT mice}

In Fig. 3a, the averaged $(n=4)$ amideCEST Z-spectrum of the WT mouse whole brain is shown for four typical $B_{1}$ values. The curve fitted using the two-step BM fitting method demonstrates the extraction of the amideCEST and the high quality of fitting. The amideCEST signals extracted by our fitting are plotted as a function of $B_{1}$ in Fig. 3 b. An exchange rate of 59.6 $\pm 9 \mathrm{~s}^{-1}$, a concentration of $41.7 \pm 7 \mathrm{mM}$ and $T_{2 \mathrm{~s}}=5.0 \pm 0.9 \mathrm{~ms}$ were extracted from the fitting.

\section{ArgCEST measurement in the GAMT ${ }^{--}$mice}

The averaged $(n=4) Z$-spectrum of the $\mathrm{GAMT}^{-/-}$mouse whole brain of is shown in Fig. 4a for the ArgCEST region. The fitted curves using the two-step group multi-B $\mathrm{B}_{1} \mathrm{BM}$ fitting method are also displayed to demonstrate the MTC background fitting results and the extraction of the ArgCEST effect. An exchange rate of $70.1 \pm 5.5 \mathrm{~s}^{-1}$, a concentration of $10.1 \pm 1.3 \mathrm{mM}$ and $T_{2 \mathrm{~s}}=$ $3.9 \pm 0.5 \mathrm{~ms}$ were extracted from the fitting for the in vivo ArgCEST. The extracted MTC background parameters $T_{2 a p p}, C_{M T C}^{0}$, and $C_{M T C}^{1}$ as a function of $B_{1}^{2}$ are plotted in Fig. $4 \mathrm{~b}-\mathrm{d}$. They can be well fitted with linear functions $\left(\mathrm{R}^{2}>0.83\right)$ and are given by

$$
\begin{gathered}
T_{2 e f f}=0.0137 B_{1}^{2}+0.0433 \\
C_{M T C}^{0}=3.0 B_{1}^{2}+0.779 \\
C_{M T C}^{1}=78.62 B_{1}^{2}+21.17
\end{gathered}
$$

The above equations were used for the following $\mathrm{B}_{1}$ dependent CrCEST and ArgCEST simulation. In Fig. 4e, the averaged ArgCEST signal extracted using two-step BM fitting with respect to the saturation power is plotted with the BM simulation curves at fixed MTC background parameters. The optimum saturation power for ArgCEST is $0.8 \mu \mathrm{T}$ according to Fig. 4e. The CrCEST signals with different exchange rates, i.e., 150, 400 and $1000 \mathrm{~s}^{-1}$ were also simulated by 
fixing the concentration at $5 \mathrm{mM}$. With exchange rates between $150-400 \mathrm{~s}^{-1}$, the curves show clear buildup and decay patterns with peaks at 1.0-1.4 $\mu \mathrm{T}$, while the signal is buildup and the signal hardly decays at a high exchange rate of $1000 \mathrm{~s}^{-1}$. To explain the $\mathrm{pH}$ dependent CEST contrasts, the ArgCEST signal as a function of exchange rate was simulated with BM equations for four typical $\mathrm{B}_{1}$ values $(0.5,1,2$ and $3 \mu \mathrm{T})$ displayed in Fig. $4 \mathrm{f}$ by fixing the MTC background.

\section{Discussion}

In this paper, we used recently developed CEST acquisition, extraction, and quantification methods to determine the exchange rates and concentrations of both amide protons and guanidinium protons in arginine residues of mobile proteins in the mouse brain. Using a $\mathrm{t}_{\mathrm{sat}}$ of $2 \mathrm{~s}$, the maximum amideCEST signal was observed with a $\mathrm{B}_{1}$ of $0.8 \mu \mathrm{T}$, comparable to the previous observed value $(1 \mu \mathrm{T})$ with a three-point method (4). The measured amide exchange rate of 59.6 $\mathrm{s}^{-1}$ was slightly higher than that measured previously by the WEX method $\left(28.6 \mathrm{~s}^{-1}\right)(27)$, but is consistent to values measured with some of the MR fingerprinting methods (34.6-47.9 $\left.\mathrm{s}^{-1}\right)(30,31)$. The consistent results from all three methods further confirm these methods are measuring the same protons, while other methods may have used acquisition parameters that are more sensitive to mixed effects of other proton pools possibly increasing the determined exchange rates.

Previous power dependent studies on both brain and muscle suggested an optimum $\mathrm{B}_{1}$ of 1.0-1.4 $\mu \mathrm{T}$ for in vivo CrCEST $(17,25,26)$, which indicates the in vivo CrCEST exchange rate is far lower than the exchange rates in solution $\left(950 \mathrm{~s}^{-1}\right)$ by comparing with simulations in Fig. 4e. The CrCEST exchange rate in tissue is estimated between 150-400 s-1 from the optimum $\mathrm{B}_{1}$ and rapid signal decay with $\mathrm{B}_{1}>1.4 \mu \mathrm{T}$ in the in vivo studies $(17,25,26)$. The $\mathrm{Cr}$ exchange rate in solution was estimated to around $1000 \mathrm{~s}^{-1}$ at room temperature and $\mathrm{pH} 7.7$ (66). The CrCEST exchange rate in the cross-linked BSA is significantly lower than that of the Cr solution (106 s $\mathrm{s}^{-1}$ vs $1000 \mathrm{~s}^{-1}$ ). The exact reason for the much lower Cr exchange rates in both tissues and crosslinked BSA is not clear. The exchange rate of $\operatorname{ArgCEST~}\left(70.1 \mathrm{~s}^{-1}\right)$ found here is far smaller than that of CrCEST in the physiological $\mathrm{pH}$ range, which should allow the selective enhancement of the CrCEST signal in the WT mouse brain by applying high $\mathrm{B}_{1}$ (Fig. 4e).

The measured guanidinium proton exchange rate determined from ArgCEST also reveals the reason behind the opposite $\mathrm{pH}$-dependence of ArgCEST signal under different conditions of $\mathrm{B}_{1}$ on mouse brain. It is known that the CEST signal increases with decreased exchange rates when 
the exchange rates are higher than the Rabi frequency of the saturation pulses used (see Fig. 4f) $(67,68)$. The guanidinium exchange rate in $\operatorname{Cr}\left(150-400 \mathrm{~s}^{-1}\right)$ is above the Rabi frequency $\left(134 \mathrm{~s}^{-1}\right)$ corresponding to $\mathrm{B}_{1}=0.5 \mu \mathrm{T}$ and should show inverse dependence on the exchange rate, i.e., $\mathrm{pH}$, as demonstrated in the Fig. 4f. On the hand, the ArgCEST (70.1 s $\left.\mathrm{s}^{-1}\right)$ is still lower than the Rabi frequency $\left(134 \mathrm{~s}^{-1}\right)$ and should be proportional to $\mathrm{pH}$ for this $\mathrm{B}_{1}$ (Fig. 4f). Therefore, the current study suggests that the inverse $\mathrm{pH}$ dependence in GuanCEST is led by the CrCEST component as the GuanCEST in WT mouse brain is a combined signal of CrCEST and ArgCEST (15). Although the protamine ArgCEST was also found to be inversely dependent on $\mathrm{pH}$ with low $\mathrm{B}_{1}(15)$, this observation cannot be used to validate the in vivo $\mathrm{pH}$ dependence due to its extremely high exchange rates as shown in the Supporting Information Figure S2. The exchange rate of Arg guanidinium protons in protamine solution is $743 \pm 20 \mathrm{~s}^{-1}$ at $\mathrm{pH} 7.0$. With high saturation $\mathrm{RF}$ strengths such as $2 \mu \mathrm{T}$, both Arg and $\mathrm{Cr}$ guanidinium exchange rates are lower than the Rabi frequency $\left(532 \mathrm{~s}^{-1}\right)$ and hence proportional to $\mathrm{pH}$. This explains the strong $\mathrm{pH}$ dependence found in mouse brain with $\mathrm{B}_{1}=2.0 \mu \mathrm{T}(20,21)$. The slow exchange rate of ArgCEST is also consistent with the observation in eggwhite at 3T, in which a clear ArgCEST peak is visible (5).

\section{Conclusion}

We used preRadCEST acquisition, two-step group BM extraction and quantification for determination of the exchange rates and concentrations of amideCEST and ArgCEST in the mouse brain. This approach can be generalized for other CEST quantitation and optimizations as well as in vivo CEST ratiometric approach for $\mathrm{pH}$ imaging.

\section{Acknowledgment}

The authors thank Drs. Dirk Isenbrandt, Robert Weiss, and Michelle Leppo for creating and providing the $\mathrm{GAMT}^{-/-}$mice.

\section{Data Availability Statement}

The code that supports the findings of this study will be made available at https://github.com/jiadixu/Two-step-Bloch-Fitting. 


\section{References:}

1. Ward K, Aletras A, Balaban RS. A new class of contrast agents for MRI based on proton chemical exchange dependent saturation transfer (CEST). J Magn Reson 2000;143(1):7987.

2. Van Zijl PC, Yadav NN. Chemical exchange saturation transfer (CEST): what is in a name and what isn't? Magn Reson Med 2011;65(4):927-948.

3. Zhou J, van Zijl PCM. Chemical exchange saturation transfer imaging and spectroscopy. Progress in Nuclear Magnetic Resonance Spectroscopy 2006;48(2-3):109-136.

4. Jin T, Wang P, Zong X, Kim S-G. MR imaging of the amide-proton transfer effect and the $\mathrm{pH}$-insensitive nuclear overhauser effect at 9.4 T. Magn Reson Med 2013;69(3):760770.

5. Sui R, Chen L, Li Y, Huang J, Chan K, Xu X, van Zijl P, Xu J. Whole-brain amide CEST imaging at $3 \mathrm{~T}$ with a steady-state radial MRI acquisition. Magn Reson Med 2021;86(2):893-906.

6. Zhou J, Heo HY, Knutsson L, van Zijl PCM, Jiang S. APT-weighted MRI: Techniques, current neuro applications, and challenging issues. J Magn Reson Imaging 2019;50(2):347-364.

7. Sun PZ, Farrar CT, Sorensen AG. Correction for artifacts induced by B(0) and B(1) field inhomogeneities in $\mathrm{pH}$-sensitive chemical exchange saturation transfer (CEST) imaging. Magn Reson Med 2007;58(6):1207-1215.

8. Sun PZ, Benner T, Kumar A, Sorensen AG. Investigation of optimizing and translating $\mathrm{pH}$-sensitive pulsed-chemical exchange saturation transfer (CEST) imaging to a 3T clinical scanner. Magn Reson Med 2008;60(4):834-841.

9. Haris M, Nanga RP, Singh A, Cai K, Kogan F, Hariharan H, Reddy R. Exchange rates of creatine kinase metabolites: feasibility of imaging creatine by chemical exchange saturation transfer MRI. NMR Biomed 2012;25(11):1305-1309.

10. Kogan F, Singh A, Cai K, Haris M, Hariharan H, Reddy R. Investigation of chemical exchange at intermediate exchange rates using a combination of chemical exchange saturation transfer (CEST) and spin-locking methods (CESTrho). Magn Reson Med 2012;68(1):107-119.

11. Kogan F, Haris M, Debrosse C, Singh A, Nanga RP, Cai K, Hariharan H, Reddy R. In vivo chemical exchange saturation transfer imaging of creatine (CrCEST) in skeletal muscle at 3T. J Magn Reson Imaging 2014;40(3):596-602.

12. Kogan F, Haris M, Singh A, Cai K, Debrosse C, Nanga RP, Hariharan H, Reddy R. Method for high-resolution imaging of creatine in vivo using chemical exchange saturation transfer. Magn Reson Med 2014;71(1):164-172.

13. Chen L, Schar M, Chan KWY, Huang J, Wei Z, Lu H, Qin Q, Weiss RG, van Zijl PCM, $\mathrm{Xu}$ J. In vivo imaging of phosphocreatine with artificial neural networks. Nat Commun 2020;11(1):1072.

14. Jiadi Xu, Lulius Juhyun Chung, Tao Jin. CEST imaging of creatine, phosphocreatine, and protein arginine residue in tissues. NMR Biomed 2022:DOI: 10.1002/nbm.4671.

15. Jin T, Wang P, Hitchens TK, Kim SG. Enhancing sensitivity of pH-weighted MRI with combination of amide and guanidyl CEST. Neuroimage 2017;157:341-350.

16. Zhang XY, Xie J, Wang F, Lin EC, Xu J, Gochberg DF, Gore JC, Zu Z. Assignment of the molecular origins of CEST signals at $2 \mathrm{ppm}$ in rat brain. Magn Reson Med 2017;78(3):881-887. 
17. Chen L, Zeng H, Xu X, Yadav NN, Cai S, Puts NA, Barker PB, Li T, Weiss RG, van Zijl $\mathrm{PCM}, \mathrm{Xu}$ J. Investigation of the contribution of total creatine to the CEST Z-spectrum of brain using a knockout mouse model. NMR Biomed 2017;30(12):e3834.

18. Zhou IY, Lu D, Ji Y, Wu L, Wang E, Cheung JS, Zhang XA, Sun PZ. Determination of multipool contributions to endogenous amide proton transfer effects in global ischemia with high spectral resolution in vivo chemical exchange saturation transfer MRI. Magn Reson Med 2019;81(1):645-652.

19. Cui J, Afzal A, Zu Z. Comparative evaluation of polynomial and Lorentzian lineshapefitted amine CEST imaging in acute ischemic stroke. Magn Reson Med 2021:https://doi.org/10.1002/mrm.29030.

20. Chen L, Van zijl P, Wei Z, Lu H, Duan W, Wong PC, Li T, Xu J. Early detection of Alzheimer's disease using creatine chemical exchange saturation transfer magnetic resonance imaging. NeuroImage 2021;236:118071.

21. Chen L, Cao S, Koehler RC, van Zijl PCM, Xu J. High-sensitivity CEST mapping using a spatiotemporal correlation-enhanced method. Magn Reson Med 2020;84(6):3342-3350.

22. Cai K, Haris M, Singh A, Kogan F, Greenberg JH, Hariharan H, Detre JA, Reddy R. Magnetic resonance imaging of glutamate. Nat Med 2012;18(2):302-306.

23. Chan KW, McMahon MT, Kato Y, Liu G, Bulte JW, Bhujwalla ZM, Artemov D, van Zij1 PC. Natural D-glucose as a biodegradable MRI contrast agent for detecting cancer. Magn Reson Med 2012;68(6):1764-1773.

24. Walker-Samuel S, Ramasawmy R, Torrealdea F, Rega M, Rajkumar V, Johnson SP, Richardson S, Goncalves M, Parkes HG, Arstad E, Thomas DL, Pedley RB, Lythgoe MF, Golay X. In vivo imaging of glucose uptake and metabolism in tumors. Nat Med 2013;19(8):1067-1072.

25. Chen L, Barker PB, Weiss RG, van Zijl PCM, Xu J. Creatine and phosphocreatine mapping of mouse skeletal muscle by a polynomial and Lorentzian line-shape fitting CEST method. Magn Reson Med 2019;81(1):69-78.

26. Chung JJ, Jin T, Lee JH, Kim SG. Chemical exchange saturation transfer imaging of phosphocreatine in the muscle. Magn Reson Med 2019;81(6):3476-3487.

27. van Zij1 PCM, Zhou J, Mori N, Payen J-F, Wilson D, Mori S. Mechanism of magnetization transfer during on-resonance water saturation. A new approach to detect mobile proteins, peptides, and lipids. Magn Reson Med 2003;49(3):440-449.

28. Yadav NN, Jones CK, Hua J, Xu J, van Zijl PC. Imaging of endogenous exchangeable proton signals in the human brain using frequency labeled exchange transfer imaging. Magn Reson Med 2013;69(4):966-973.

29. Heo HY, Han Z, Jiang S, Schar M, van Zijl PCM, Zhou J. Quantifying amide proton exchange rate and concentration in chemical exchange saturation transfer imaging of the human brain. Neuroimage 2019;189:202-213.

30. Perlman O, Ito H, Herz K, Shono N, Nakashima H, Zaiss M, Chiocca EA, Cohen O, Rosen MS, Farrar CT. Quantitative imaging of apoptosis following oncolytic virotherapy by magnetic resonance fingerprinting aided by deep learning. Nature Biomedical Engineering 2021:https://oi.org/10.1038/s41551-41021-00809-41557.

31. Cohen O, Huang S, McMahon MT, Rosen MS, Farrar CT. Rapid and quantitative chemical exchange saturation transfer (CEST) imaging with magnetic resonance fingerprinting (MRF). Magn Reson Med 2018;80(6):2449-2463. 
32. Liu J, de Zwart JA, van Gelderen P, Murphy-Boesch J, Duyn JH. Effect of head motion on MRI B0 field distribution. Magn Reson Med 2018.

33. Wen J, Cross AH, Yablonskiy DA. On the role of physiological fluctuations in quantitative gradient echo MRI: implications for GEPCI, QSM, and SWI. Magn Reson Med 2015;73(1):195-203.

34. Chen L, Wei Z, Cai S, Li Y, Liu G, Lu H, Weiss RG, van Zij1 PCM, Xu J. Highresolution creatine mapping of mouse brain at $11.7 \mathrm{~T}$ using non-steady-state chemical exchange saturation transfer. NMR Biomed 2019;32(11):e4168.

35. Chen L, Wei Z, Chan KWY, Cai S, Liu G, Lu H, Wong PC, van Zijl PCM, Li T, Xu J. Protein aggregation linked to Alzheimer's disease revealed by saturation transfer MRI. Neuroimage 2018;188:380-390.

36. Zhou Y, van Zij1 PCM, Xu X, Xu J, Li Y, Chen L, Yadav NN. Magnetic resonance imaging of glycogen using its magnetic coupling with water. Proc Natl Acad Sci U S A 2020;117(6):3144-3149.

37. Du J, Takahashi AM, Bydder M, Chung CB, Bydder GM. Ultrashort TE imaging with off-resonance saturation contrast (UTE-OSC). Magn Reson Med 2009;62(2):527-531.

38. Liu R, Zhang H, Niu W, Lai C, Ding Q, Chen W, Liang S, Zhou J, Wu D, Zhang Y. Improved chemical exchange saturation transfer imaging with real-time frequency drift correction. Magn Reson Med 2019;81(5):2915-2923.

39. Windschuh J, Zaiss M, Ehses P, Lee JS, Jerschow A, Regatte RR. Assessment of frequency drift on CEST MRI and dynamic correction: application to gagCEST at $7 \mathrm{~T}$. Magn Reson Med 2019;81(1):573-582.

40. Simegn GL, Van der Kouwe AJW, Robertson FC, Meintjes EM, Alhamud A. Real-time simultaneous shim and motion measurement and correction in glycoCEST MRI using double volumetric navigators (DvNavs). Magn Reson Med 2019;81(4):2600-2613.

41. Cai K, Singh A, Poptani H, Li W, Yang S, Lu Y, Hariharan H, Zhou XJ, Reddy R. CEST signal at 2ppm (CEST@2ppm) from Z-spectral fitting correlates with creatine distribution in brain tumor. NMR Biomed 2015;28(1):1-8.

42. Desmond KL, Moosvi F, Stanisz GJ. Mapping of amide, amine, and aliphatic peaks in the CEST spectra of murine xenografts at 7 T. Magn Reson Med 2014;71(5):1841-1853.

43. Zaiss M, Windschuh J, Goerke S, Paech D, Meissner JE, Burth S, Kickingereder P, Wick W, Bendszus M, Schlemmer HP, Ladd ME, Bachert P, Radbruch A. Downfield-NOEsuppressed amide-CEST-MRI at 7 Tesla provides a unique contrast in human glioblastoma. Magn Reson Med 2016.

44. Zhou IY, Wang E, Cheung JS, Zhang X, Fulci G, Sun PZ. Quantitative chemical exchange saturation transfer (CEST) MRI of glioma using Image Downsampling Expedited Adaptive Least-squares (IDEAL) fitting. Scientific Reports 2017;7(1):84.

45. Mueller S, Stirnberg R, Akbey S, Ehses P, Scheffler K, Stocker T, Zaiss M. Whole brain snapshot CEST at 3T using 3D-EPI: Aiming for speed, volume, and homogeneity. Magn Reson Med 2020;84(5):2469-2483.

46. Renema WK, Schmidt A, van Asten JJ, Oerlemans F, Ullrich K, Wieringa B, Isbrandt D, Heerschap A. MR spectroscopy of muscle and brain in guanidinoacetate methyltransferase (GAMT)-deficient mice: validation of an animal model to study creatine deficiency. Magn Reson Med 2003;50(5):936-943. 
47. Kan HE, Meeuwissen E, van Asten JJ, Veltien A, Isbrandt D, Heerschap A. Creatine uptake in brain and skeletal muscle of mice lacking guanidinoacetate methyltransferase assessed by magnetic resonance spectroscopy. J Appl Physiol 2007;102(6):2121-2127.

48. McMahon MT, Gilad AA, Zhou J, Sun PZ, Bulte JWM, van Zijl PCM. Quantifying exchange rates in chemical exchange saturation transfer agents using the saturation time and saturation power dependencies of the magnetization transfer effect on the magnetic resonance imaging signal (QUEST and QUESP): Ph calibration for poly-L-lysine and a starburst dendrimer. Magn Reson Med 2006;55(4):836-847.

49. Zaiss M, Angelovski G, Demetriou E, McMahon MT, Golay X, Scheffler K. QUESP and QUEST revisited - fast and accurate quantitative CEST experiments. Magn Reson Med 2018;79(3):1708-1721.

50. Wu R, Xiao G, Zhou IY, Ran C, Sun PZ. Quantitative chemical exchange saturation transfer (qCEST) MRI - omega plot analysis of RF-spillover-corrected inverse CEST ratio asymmetry for simultaneous determination of labile proton ratio and exchange rate. NMR Biomed 2015;28(3):376-383.

51. Meissner JE, Goerke S, Rerich E, Klika KD, Radbruch A, Ladd ME, Bachert P, Zaiss M. Quantitative pulsed CEST-MRI using Omega-plots. NMR Biomed 2015;28(10):11961208.

52. Sun PZ. Quasi-steady-state CEST (QUASS CEST) solution improves the accuracy of CEST quantification: QUASS CEST MRI-based omega plot analysis. Magn Reson Med 2021;86(2):765-776.

53. Dixon WT, Hancu I, Ratnakar SJ, Sherry AD, Lenkinski RE, Alsop DC. A multislice gradient echo pulse sequence for CEST imaging. Magn Reson Med 2010;63(1):253-256.

54. Henkelman RM, Huang X, Xiang QS, Stanisz GJ, Swanson SD, Bronskill MJ. Quantitative interpretation of magnetization transfer. Magn Reson Med 1993;29(6):759766.

55. Li JG, Graham SJ, Henkelman RM. A Flexible Magnetization Transfer Line Shape Derived from Tissue Experimental Data. Magn Reson Med 1997;37(13):866-871.

56. Wilhelm MJ, Ong HH, Wehrli SL, Li C, Tsai PH, Hackney DB, Wehrli FW. Direct magnetic resonance detection of myelin and prospects for quantitative imaging of myelin density. Proc Natl Acad Sci USA 2012;109(24):9605-9610.

57. Xu J, Chan KW, Xu X, Yadav N, Liu G, van Zijl PC. On-resonance variable delay multipulse scheme for imaging of fast-exchanging protons and semisolid macromolecules. Magn Reson Med 2017;77(2):730-739.

58. Chen L, Xu X, Zeng H, Chan K, Yadav NN, Cai S, Schunke KJ, Faraday N, van Zij1 PCM, Xu J. Separating Fast and Slow Exchange Transfer and Magnetization Transfer Using Off-resonance Variable Delay Multiple Pulse (VDMP) MRI. Magn Reson Med 2018;80(4):1568-1576.

59. Gochberg DF, Gore JC. Quantitative imaging of magnetization transfer using an inversion recovery sequence. Magn Reson Med 2003;49(3):501-505.

60. van Gelderen P, Jiang X, Duyn JH. Rapid measurement of brain macromolecular proton fraction with transient saturation transfer MRI. Magn Reson Med 2017;77(6):2174-2185.

61. Henkelman RM, Stanisz GJ, Graham SJ. Magnetization transfer in MRI: a review. NMR Biomed 2001;14(2):57-64.

62. Graham SJ, Henkelman RM. Pulsed magnetization transfer imaging: evaluation of technique. Radiology 1999;212(3):903-910. 
63. Sled JG, Pike GB. Quantitative Interpretation of Magnetization Transfer in Spoiled Gradient Echo MRI Sequences. J Magn Reson 2000;145(1):24-36.

64. GB P. Pulsed magnetization transfer contrast in gradient echo imaging: a two-pool analylic description of signal response. . Magn Reson Med 1996;36:95-103.

65. Levesque IR, Pike GB. Characterizing healthy and diseased white matter using quantitative magnetization transfer and multicomponent $\mathrm{T}(2)$ relaxometry: A unified view via a four-pool model. Magn Reson Med 2009;62(6):1487-1496.

66. Goerke S, Zaiss M, Bachert P. Characterization of creatine guanidinium proton exchange by water-exchange (WEX) spectroscopy for absolute-pH CEST imaging in vitro. NMR Biomed 2014;27(5):507-518.

67. Jin T, Kim SG. Approximated analytical characterization of the steady-state chemical exchange saturation transfer (CEST) signals. Magn Reson Med 2019;82(5):1876-1889.

68. Zong X, Wang P, Kim SG, Jin T. Sensitivity and source of amine-proton exchange and amide-proton transfer magnetic resonance imaging in cerebral ischemia. Magn Reson Med 2014;71(1):118-132.

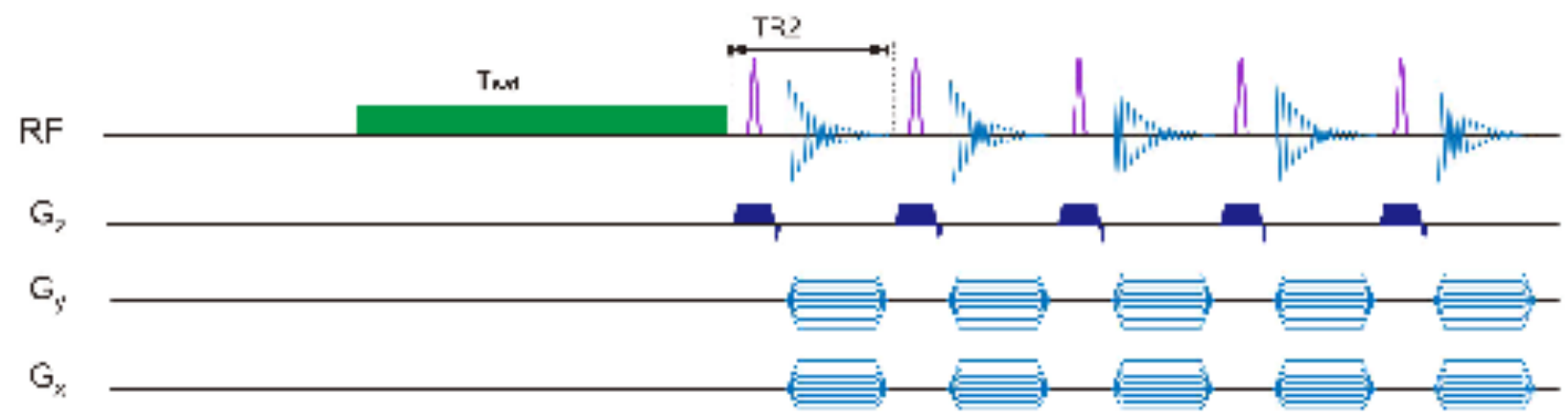

Figure 1. (a) Timing diagram of the preRadCEST sequence. $T_{\text {sat }}$ is the length of the CW saturation period, while TR2 is the time duration between successive readout pules. 

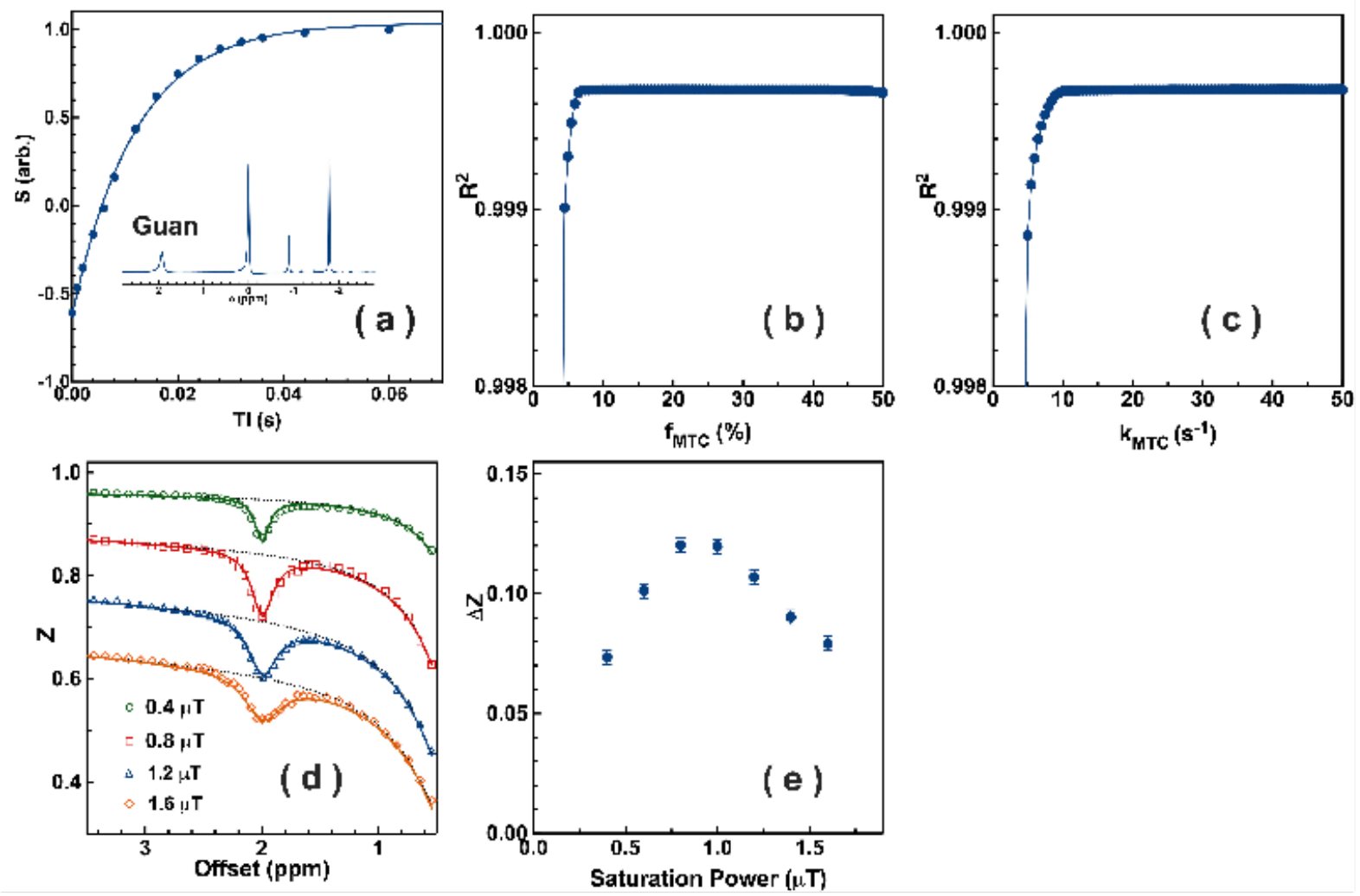

Figure 2. (a) The inversion recovery curve of peaks at $1.92 \mathrm{ppm}$ (Guan) of Cr in 15\% cross-linked BSA at room temperature and $\mathrm{pH}$ 7.7. The curve was fitted using a single exponential curve (Supporting Information Eq. S1). Inset: typical 1D NMR spectrum of CrCrossBSA phantom. (bd) The impact of different (b) $f_{\mathrm{MTC}}$ and (c) $k_{\mathrm{MTC}}$ on the MTC background fitting. The goodness of the fitting results was evaluated by the $\mathrm{R}^{2}$ coefficient of determination. (b) $k_{\mathrm{MTC}}=20 \mathrm{~s}^{-1}$ set for the simulation (c) In the $k_{\mathrm{MTC}}$ simulation, $f_{\mathrm{MTC}}=10 \%$. (d) Typical Z-spectra of the GuanCEST region recorded with a $\mathrm{B}_{1}$ of $0.4,0.8,1.2$ and $1.6 \mu \mathrm{T}$ and an illustration of the two-step multi- $\mathrm{B}_{1} \mathrm{BM}$ fitting scheme for extracting and quantifying the GuanCEST signal $\left(\mathrm{R}^{2}=0.995\right)$. All CrCrossBSA experiments were performed at room temperature. The black dashed lines are the background Zspectrum, while the solid lines are fitted CrCEST curves with the fixed water and MTC background. In the whole fitting, the measured water $T_{l w}=1.8 \mathrm{~s}$ was used, while $T_{1 \mathrm{MTC}}=1 \mathrm{~s}$ and $T_{2 \mathrm{MTC}}=10 \mu \mathrm{s}$ for the MTC pool were fixed. (f) The $\mathrm{B}_{1}$-dependent CrCEST values for the CrCrossBSA phantom extracted with the two-step multi-B $\mathrm{B}_{1} \mathrm{BM}$ fitting. 

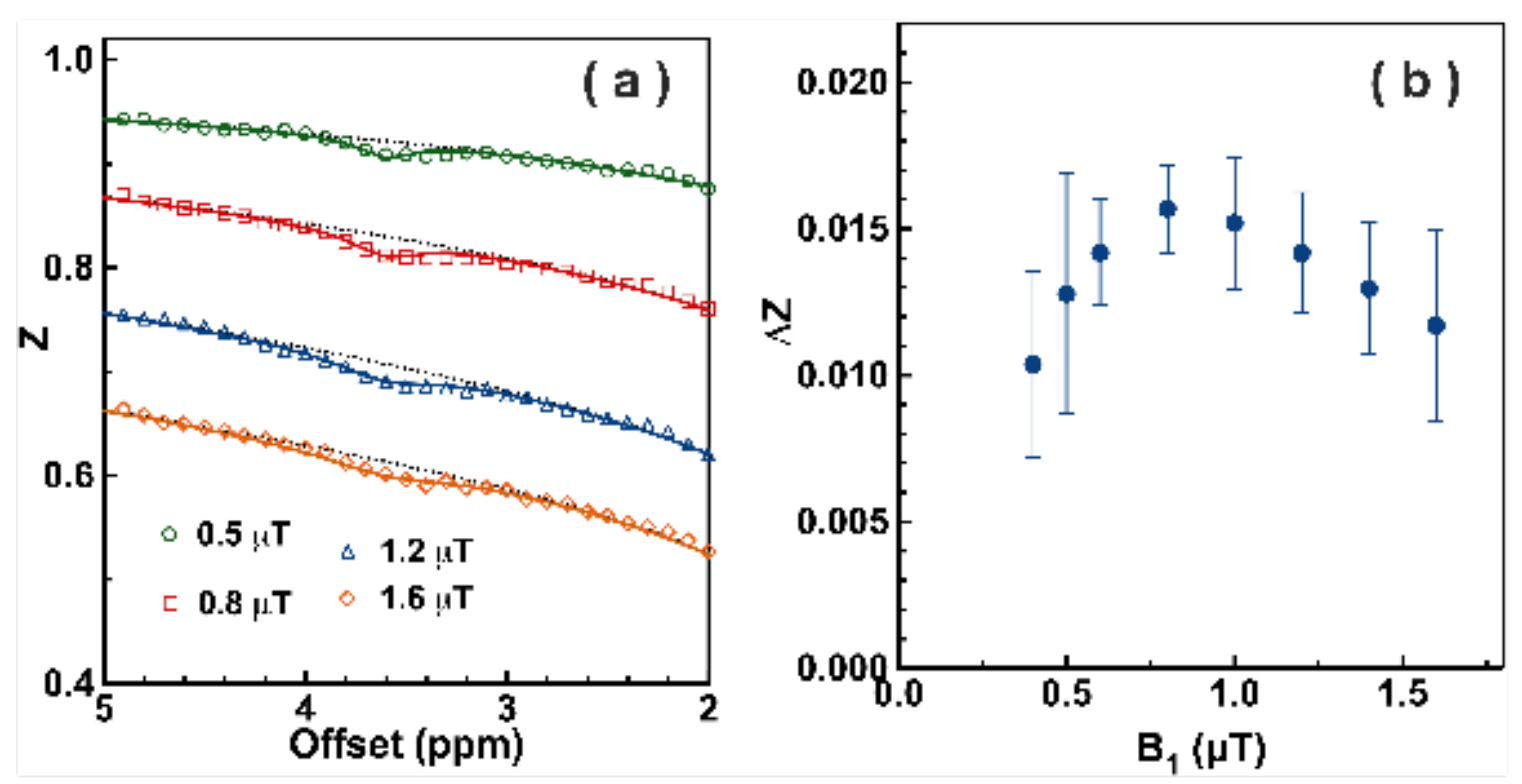

Figure 3. (a) Averaged Z-spectrum of the amideCEST $(n=4)$ for the WT mice recorded using preRadCEST with a fixed saturation length of $5 \mathrm{~s}$ and varying $\mathrm{B}_{1}=0.5,0.8,1.2$ and $1.6 \mu \mathrm{T}$ together with the two-step B1 group fitting curves. Dashed lines are the MTC background fitting curves, while solid lines are the difference Z-spectrum fitting. (b) The extracted amideCEST signal $(\Delta \mathrm{Z})$ of the WT whole brain as a function of $\mathrm{B}_{1}$ extracted with the two-step BM fitting. 

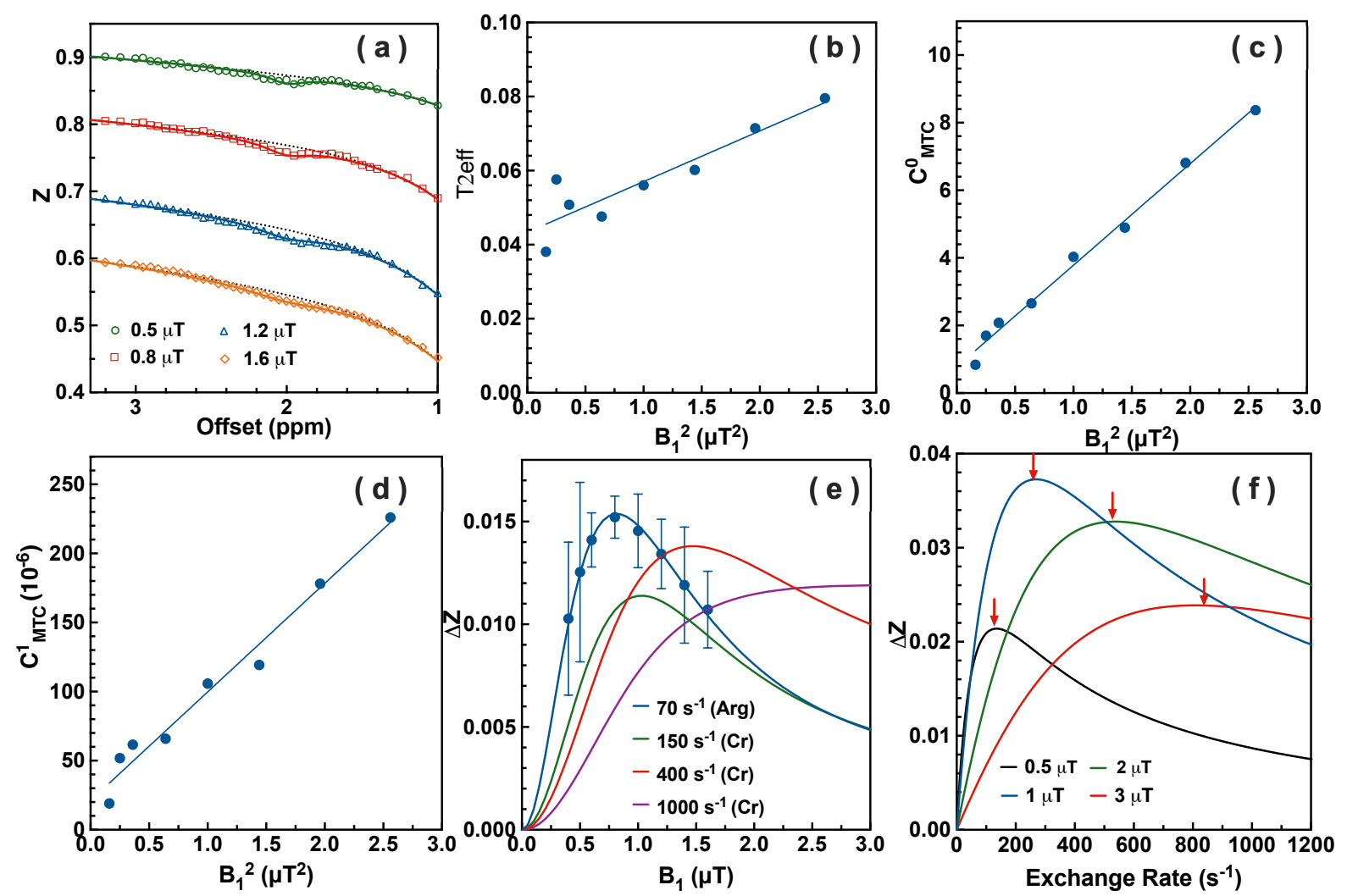

Figure 4. (a) Averaged Z-spectrum of the ArgCEST $(n=4)$ for the $\mathrm{GAMT}^{-/-}$mice recorded with four typical $\mathrm{B}_{1}$ values together with the two-step BM fitting curves. Dashed lines are the MTC background fitting curves, while solid-lines are the difference Z-spectrum fitting. (b-c) The extracted MTC background parameters (b) $T_{2 a p p}$, (c) $C_{M T C}^{0}$, and (d) $C_{M T C}^{1}$ as a function of $B_{1}^{2}$. Solid lines are the linear fitting curves. (e) The averaged saturation power-dependent ArgCEST values at $2 \mathrm{ppm}$ for the whole brain of $\mathrm{GAMT}^{-/-}$mouse $(\mathrm{n}=4)$. The solid lines are the simulated curves for both Arg and Cr GuanCEST with different exchange rates and concentrations, i.e., $70.0 \mathrm{~s}^{-1}(10.0$ $\mathrm{mM})$ for Arg, $150 \mathrm{~s}^{-1}(5 \mathrm{mM}), 400 \mathrm{~s}^{-1}(5 \mathrm{mM})$ and $1000 \mathrm{~s}^{-1}(5 \mathrm{mM})$ for Cr. In the fitting, $T_{2 \mathrm{~s}}=3.9$ $\mathrm{ms}, T_{1 \mathrm{w}}=1.8 \mathrm{~s}, T_{1 \mathrm{MTC}}=1 \mathrm{~s}, T_{2 \mathrm{MTC}}=10 \mu \mathrm{s}, f_{\mathrm{MTC}}=10 \%$, saturation time $5 \mathrm{~s}$ was fixed. (f) The simulated exchange rate dependent GuanCEST signal for four typical saturation powers, i.e., $0.5 \mu \mathrm{T}, 1 \mu \mathrm{T}$, $2 \mu \mathrm{T}$ and $3 \mu \mathrm{T}$. The Rabi frequencies are labeled with red arrows, i.e. $134 \mathrm{~s}^{-1}(0.5 \mu \mathrm{T}), 268 \mathrm{~s}^{-1}(1$ $\mu \mathrm{T}), 538 \mathrm{~s}^{-1}(2 \mu \mathrm{T}), 806 \mathrm{~s}^{-1}(3 \mu \mathrm{T})$. In the fitting, $f_{\text {guan }}=11.9 \mathrm{mM}$, saturation time $5 \mathrm{~s}$ and $T_{2 \text { guan }}=15$ ms were used. 


\section{Supporting Information:}

\section{Bloch-McConnell (BM) Simulations:}

The observed MTC/CEST signal under influence of a continuous RF pulse can be calculated using the coupled Bloch equations for a three-pool model: water $\left(M_{x w}, M_{y w}, M_{z w}\right), \operatorname{CEST}\left(M_{x s}, M_{y s}, M_{z s}\right)$ and $\operatorname{MTC}\left(M_{z M T C}\right)(1): M T C$

$$
\begin{gathered}
\mathrm{d} \vec{M}=\Lambda \vec{M} \\
\vec{M}=\left[\begin{array}{c}
M_{x w} \\
M_{y w} \\
M_{z w} \\
M_{x s} \\
M_{y s} \\
M_{z s} \\
M_{z M T C}
\end{array}\right]
\end{gathered}
$$

Here, the transverse components of MTC $\left(M_{x M T C}, M_{y M T C}\right)$ are set to zero due to the extremely short $T_{2}$ in the semisolid.

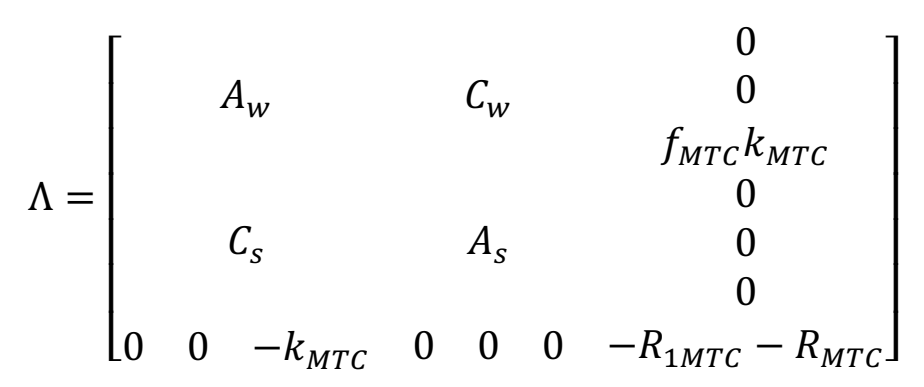

$A_{w}$ and $A_{s}$ are two $3 \times 3$ matrixes that describe the evolution of the water and solute magnetizations under an RF pulse, respectively, while $C_{w}$ and $C_{s}$ are two $3 \times 3$ matrixes describing the chemical exchange processes from the water and from the solute pools, respectively. The exchange between solute pool and MTC pool was neglected. $k_{M T C}$ is the exchange rate from the MTC pool to water, and $f_{m t c}$ is the fractional concentration of the MTC protons to the water protons. $R_{1 M T C}$ is the longitudinal relaxation rate of the MTC pool. $R_{M T C}$ is the lineshape of the macromolecular pool together with other saturation transfer components. The MTC lineshape has been reported as Gaussian for Agar and Super-Lorentzian for semi-solid biological tissues $(2,3)$. Considering the frequency range studies in Z-spectra is far smaller than those in MTC studies and the complicated 
contributions from other exchangeable protons, the MTC lineshape is assumed to be a linear function:

$$
R_{M T C}=C_{M T C}^{0}+2 \pi\left(\Delta-\Delta_{M T C}\right) C_{M T C}^{1}
$$

where $\Delta_{M T C}$ is the offset of the MTC pool resonance center relative to the water signal (Hz). $A_{w}$ and $A_{s}$ are given by

$$
\begin{aligned}
A_{w} & =\left[\begin{array}{ccc}
-R_{2 w} & 2 \pi \Delta_{w} & 0 \\
-2 \pi \Delta_{w} & -R_{2 w} & \gamma B_{1} \\
0 & -\gamma B_{1} & -R_{1 w}
\end{array}\right] \\
A_{s} & =\left[\begin{array}{ccc}
-R_{2 s} & 2 \pi \Delta_{s} & 0 \\
-2 \pi \Delta_{s} & -R_{2 s} & \gamma B_{1} \\
0 & -\gamma B_{1} & -R_{1 s}
\end{array}\right]
\end{aligned}
$$

where $R_{2 w}$ and $R_{2 s}$ are the transverse relaxation rates and $R_{1 w}$ and $R_{1 s}$ the longitudinal relaxation rates for the two pools. $\Delta_{w}$ and $\Delta_{s}$ are chemical shift offsets $(\mathrm{Hz})$ for the two pools relative to the water proton pool. The exchange matrixes $C_{w}$ and $C_{s}$ are given by

$$
\begin{gathered}
C_{w}=\left[\begin{array}{cccc}
f_{s} & k_{s} & 0 & 0 \\
0 & f_{s} & k_{s} & 0 \\
0 & 0 & f_{s} & k_{s}
\end{array}\right] \\
C_{s}=\left[\begin{array}{ccc}
-k_{s} & 0 & 0 \\
0 & -k_{s} & 0 \\
0 & 0 & -k_{s}
\end{array}\right]
\end{gathered}
$$

\section{Exchange rate measurement of the CrCEST:}

The exchange rates of CrCEST in the cross-linked BSA phantoms were measured by the magnetization recovery of the exchangeable proton peaks after inversion by a single selective Gaussian pulse (width of $2.5 \mathrm{~ms}$ ) assumed to have negligible effect on the semisolid. After a delay TI, the NMR signal was detected by a 3-9-19 WATER suppression by GrAdient Tailored Excitation (WATERGATE) sequence with a total echo time of $1 \mathrm{~ms}$. Repetition time was $10 \mathrm{~s}$. A list of 15 inversion times from $0 \mathrm{~ms}$ to $200 \mathrm{~ms}$ were used. The offset was set to $2.0 \mathrm{ppm}$ (with respect to water). The recovery rate was determined by the apparent relaxation rate, $1 / T_{1}+k_{s w} \approx$ $k_{s w}$, from the fitting of the recovery curve using the following equation

$$
S=A-B e^{-k_{s w} T I}
$$




\section{In vivo MRS on GAMT ${ }^{-/-}$mice:}

The total creatine $(\mathrm{Cr}+\mathrm{PCr})$ concentrations in all GAMT-/- mouse brains were obtained from in vivo proton MRS experiments performed on voxels of $2.5 \times 2.5 \times 2.5 \mathrm{~mm}^{3}$ each using a stimulated echo acquisition mode $(\mathrm{STEAM})$ sequence $(\mathrm{TE}=3 \mathrm{~ms}, \mathrm{TM}=10 \mathrm{~ms}, \mathrm{TR}=2.5 \mathrm{~s}, \mathrm{NA}$ =64). These MRS spectra for the four GAMT ${ }^{-/}$are plotted below and were fitted with the LCModel as detailed previously (4), showing negligible total $\mathrm{Cr}(1.47 \pm 0.68 \mathrm{mM})$ :
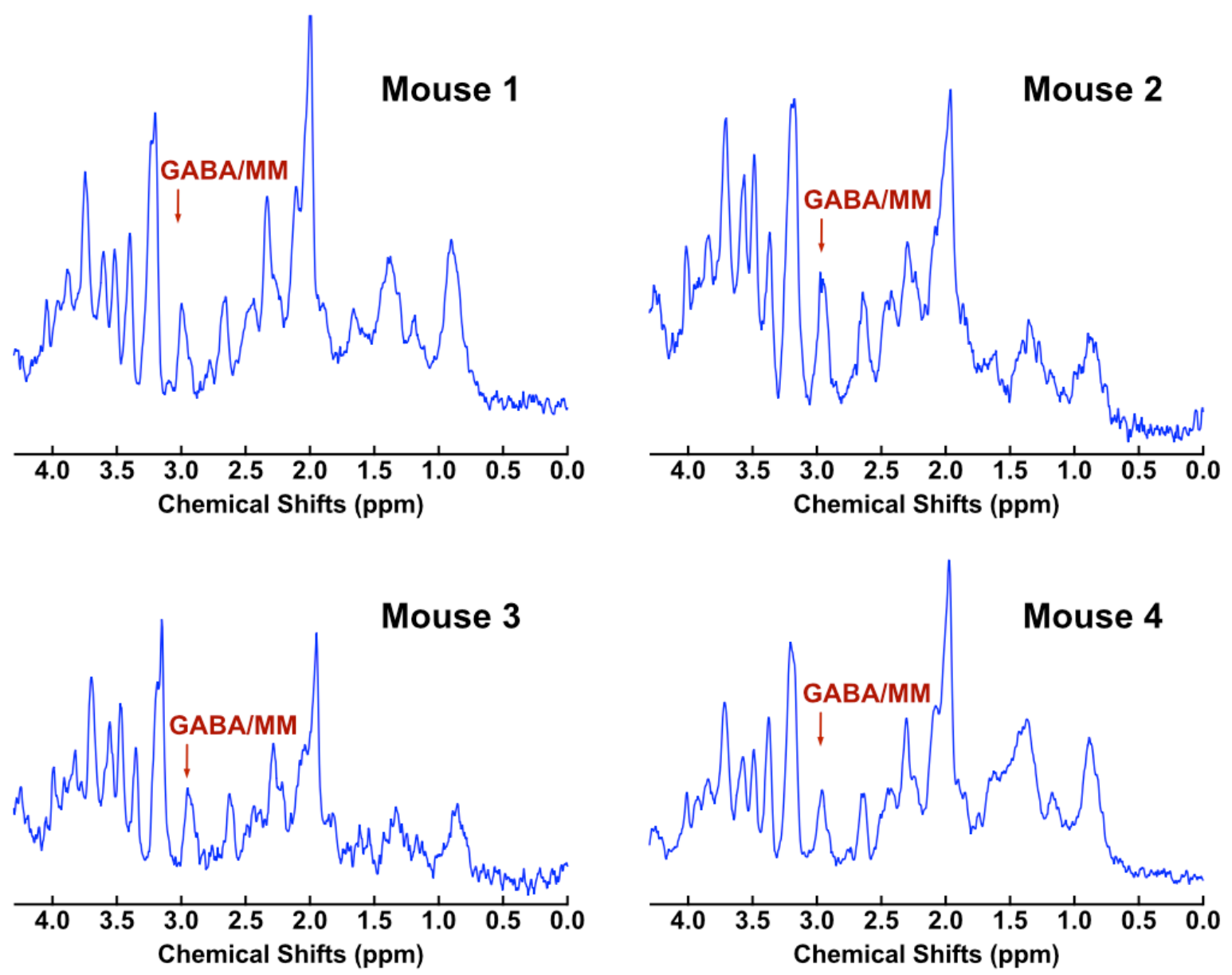

Supporting Information Figure S1: in vivo ${ }^{1} \mathrm{H}$ NMR spectra measured from regions of the midbrain in all four GAMT ${ }^{-/-}$mice. The peak at 3 ppm of all $\mathrm{GAMT}^{-/-}$mice shows a residual signal due to gamma aminobutyric acid (GABA) and mobile macromolecules (MM), while LCModel fitting give negligible $\mathrm{Cr} / \mathrm{PCr}$ remaining.

\section{Bloch-McConnell (BM) Fitting results:}


Table S1: The $T_{2 \text { app }}, C_{M T C}^{0}$ and $C_{M T C}^{1}$ obtained by the MTC background fitting for the CrCrossBSA phantom. $T_{1 \mathrm{w}}=1.8 \mathrm{~s}, T_{1 \mathrm{MTC}}=1 \mathrm{~s}, k_{\mathrm{MTC}}=20 \mathrm{~s}^{-1}$ and $f_{M T C}=10 \%$ were fixed during the global BM fitting.

\begin{tabular}{l|ccc}
$B_{1}(\mu \mathrm{T})$ & $T_{2 a p p}(\mathrm{~s})$ & $C_{M T C}^{0}\left(s^{-1}\right)$ & $C_{M T C}^{1}\left(10^{-6} s^{-1} / \mathrm{rad}\right)$ \\
\hline 0.4 & 0.0585 & 0.677 & 19.3101 \\
0.6 & 0.0605 & 0.775 & 11.623 \\
0.8 & 0.0648 & 1.366 & 15.504 \\
1.0 & 0.0732 & 2.482 & 42.6371 \\
1.2 & 0.0862 & 4.143 & 86.290 \\
1.4 & 0.0994 & 5.461 & 106.096 \\
1.6 & 0.1089 & 7.073 & 139.772
\end{tabular}

Table S2: The $T_{2 \text { app, }} C_{M T C}^{0}$ and $C_{M T C}^{1}$ obtained by the MTC and water background fitting for in vivo WT mice. $T_{1 \mathrm{w}}=1.8 \mathrm{~s}, T_{1 \mathrm{MTC}}=1 \mathrm{~s}, k_{\mathrm{MTC}}=20 \mathrm{~s}^{-1}$ and $f_{M T C}=10 \%$ were fixed during the global BM fitting.

\begin{tabular}{c|ccc}
$B_{1}(\mu \mathrm{T})$ & $T_{2 a p p}(\mathrm{~s})$ & $C_{M T C}^{0}\left(s^{-1}\right)$ & $C_{M T C}^{1}\left(10^{-6} s^{-1} / \mathrm{rad}\right) f$ \\
\hline 0.4 & 0.0499 & 0.8106 & 24.920 \\
0.5 & 0.0173 & 1.118 & 33.726 \\
0.6 & 0.0285 & 1.803 & 51.731 \\
0.8 & 0.0298 & 2.898 & 86.045 \\
1.0 & 0.0303 & 4.119 & 118.428 \\
1.2 & 0.0310 & 5.173 & 145.000 \\
1.4 & 0.0309 & 6.173 & 170.193 \\
1.6 & 0.0382 & 7.592 & 207.347
\end{tabular}

Table S3: The $T_{2 \text { app }}, C_{M T C}^{0}$ and $C_{M T C}^{1}$ obtained by the MTC background fitting for in vivo GAMT $^{-}$ ${ }^{1-}$ mice. $T_{1 \mathrm{w}}=1.8 \mathrm{~s}, T_{1 \mathrm{MTC}}=1 \mathrm{~s}, k_{\mathrm{MTC}}=20 \mathrm{~s}^{-1}$ and $f_{M T C}=10 \%$ were fixed during the global BM fitting.

\begin{tabular}{c|ccc}
$B_{1}(\mu \mathrm{T})$ & $T_{2 a p p}(\mathrm{~s})$ & $C_{M T C}^{0}\left(\mathrm{~s}^{-1}\right)$ & $C_{M T C}^{1}\left(10^{-6} \mathrm{~s}^{-1} / \mathrm{rad}\right)$ \\
\hline 0.4 & 0.0380 & 0.836 & 18.992 \\
0.5 & 0.0575 & 1.695 & 51.852 \\
0.6 & 0.0508 & 2.080 & 61.582 \\
0.8 & 0.0475 & 2.653 & 65.883 \\
1.0 & 0.0560 & 4.032 & 105.768 \\
1.2 & 0.0601 & 4.893 & 119.264 \\
1.4 & 0.0714 & 6.810 & 178.059 \\
1.6 & 0.0795 & 8.370 & 225.996
\end{tabular}




\section{Arg and Amide exchange rates in protamine:}

Protamine has been used previously as model protein for an ArgCEST study (5). Therefore, the exchange rates of guanidinium protons were measured in the protamine solution for comparison with in vivo results. Several $50 \mathrm{mg} / \mathrm{ml}$ protamine $\left(1.1 \times 10^{4} \mathrm{mM}\right)$ solutions (SigmaAldrich) were prepared in phosphate buffered saline (PBS) and titrated to $\mathrm{pH} 6.4 \pm 0.1,6.7 \pm 0.1$, $7.0 \pm 0.1$, and $7.3 \pm 0.1$. The protamine solutions were studied in $5 \mathrm{~mm} \mathrm{NMR}$ glass tubes at $37{ }^{\circ} \mathrm{C}$ at 17.6T. The exchange rates of the amide and ArgCEST in protamine were measured with selective inversion recovery method detailed in the main text method section. A list of inversion times (start $=0 \mathrm{~ms}$, end $=60 \mathrm{~ms}$, number of samples $=15$ ) was used for the ArgCEST exchange rate measurement in protamine. The offsets (with respect to water) were set to $3.5 \mathrm{ppm}$ for amideCEST and $2.0 \mathrm{ppm}$ for ArgCEST.

17.6T NMR spectra of protamine for typical $\mathrm{pH}$ value are presented in Fig. S2, presenting both amide (3.5 ppm) and Guan (2.0 ppm) exchanging proton groups. Due to the abundant Arg residues in protamine (6), aromatic and other overlapping protons were assumed to be negligible. The Guan peak broadens significantly at $\mathrm{pH} 7.3$, due to the increased exchange rate. Typical inversion recovery curves for the amide and Guan peaks are shown in Fig. S2b, together with the curves fitted using Eq. S1. The $\mathrm{pH}$ dependence of the exchange rates for the amide and Guan groups are presented in Fig. S2c. At pH 7.0, $k_{\text {amide }}=112 \pm 5 \mathrm{~s}^{-1}$ and $k_{\text {Guan }}=743 \pm 20 \mathrm{~s}^{-1}$ were determined. Beyond that, the guan exchange rate is difficult to determine due to the extremely high value, since its saturation/inversion in the selective inversion method will be an obstacle.
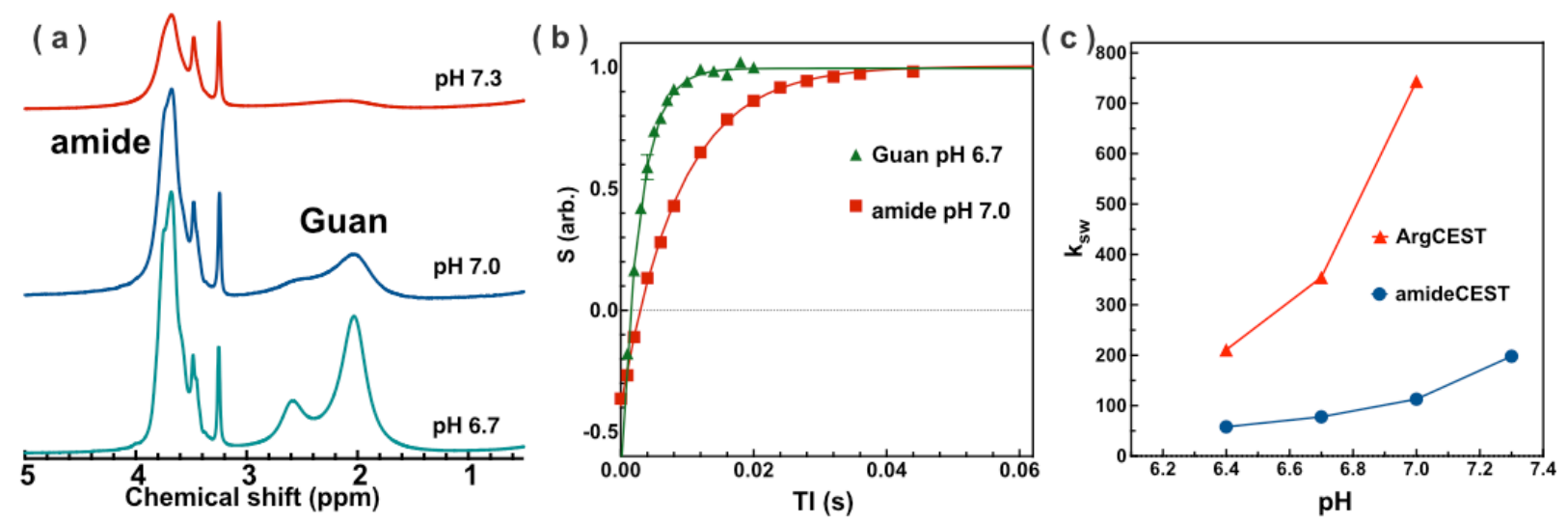

Supporting Information Figure S2: (a) The NMR spectra of protamine solution recorded with a WATERGATE sequence $(\mathrm{TE}=1 \mathrm{~ms})$ at three $\mathrm{pH}$ values $(6.7,7.0$ and 7.3$)$. (b) The averaged 
inversion recovery curves $(\mathrm{n}=3)$ of peaks at $2 \mathrm{ppm}$ (Guan) and $3.5 \mathrm{ppm}$ (amide) in protamine solution, respectively. The curves were fitted using a single exponential curve (Eq. 1). (c) pH dependence of the exchange rates for the ArgCEST and amideCEST peaks. All protamine experiments were performed at $37^{\circ} \mathrm{C}$.

\section{References:}

1. Ropele S, Seifert T, Enzinger C, Fazekas F. Method for quantitative imaging of the macromolecular 1H fraction in tissues. Magn Reson Med 2003;49(5):864-871.

2. Li JG, Graham SJ, Henkelman RM. A Flexible Magnetization Transfer Line Shape Derived from Tissue Experimental Data. Magn Reson Med 1997;37(13):866-871.

3. Wilhelm MJ, Ong HH, Wehrli SL, Li C, Tsai PH, Hackney DB, Wehrli FW. Direct magnetic resonance detection of myelin and prospects for quantitative imaging of myelin density. Proc Natl Acad Sci USA 2012;109(24):9605-9610.

4. Chen L, Zeng H, Xu X, Yadav NN, Cai S, Puts NA, Barker PB, Li T, Weiss RG, van Zijl $\mathrm{PCM}, \mathrm{Xu}$ J. Investigation of the contribution of total creatine to the CEST Z-spectrum of brain using a knockout mouse model. NMR Biomed 2017;30(12):e3834.

5. Jin T, Wang P, Hitchens TK, Kim SG. Enhancing sensitivity of pH-weighted MRI with combination of amide and guanidyl CEST. Neuroimage 2017;157:341-350.

6. Husebo S, Tausjo J. [Patients dying in institutions. A medical and human challenge]. Tidsskr Nor Laegeforen 1990;110(17):2233-2235. 